\title{
Anregungen zur Darstellung von Routineformeln in einsprachigen Lernerwörterbüchern für DaF
}

\author{
Silke Lipinski (Leipzig)
}

\begin{abstract}
Routine formulas (RFs) carry many functions in a language system. Thus, not only knowing them but also mastering their use is of major importance for the L2-learner. For this reason, it is important to also describe RFs adequately in learners' dictionaries. However, as previous research shows, there is a major discrepancy between lexicographical requirements and reality. Some demands for the way in which RFs should be presented can be derived from the list of criteria which are used to evaluate dictionary entries of RFs. Some of these requirements may be called phraseographical minima, the realization of some other requirements that have yet to be developed in detail. This article intends to show options on how to improve the lexicography of RFs in print dictionaries for L2-learners. Suggestions for the presentation of RFs in the lexicographical macro-, micro-, and medio-structure are made and discussed.
\end{abstract}

\section{$1 \quad$ Einleitung}

Die Anzahl an Publikationen zum Stellenwert von phraseologischen Einheiten innerhalb des Sprachsystems ist in den letzten Jahren stetig gewachsen. Mit der zunehmenden Beachtung phraseologischer Einheiten wuchs auch die Wahrnehmung ihrer Relevanz für den Fremdsprachenlerner und im Zuge dessen das Interesse an der adäquaten Darstellung dieses Phänomens in Lernerwörterbüchern. Für verschiedene phraseologische Subgruppen wurde die lernerlexikografische Darstellungspraxis untersucht und beschrieben. So geschehen auch die für die Subgruppe der Routineformeln (RF), welche hier im Zentrum der Überlegungen stehen wird.

Unter RF sind kommunikative Phraseologismen, die Funktionen bei der Herstellung, der Definition, dem Vollzug und der Beendigung immer wiederkehrender kommunikativer Handlungen (Burger 2007: 36) einnehmen, zu verstehen. Sie definieren sich in erster Linie durch ihre pragmatische Festigkeit (cf. Stein 2004: 264). RF stellen zudem situations- und institutionsgebundene Formeln dar, wie z. B. Kein Problem!, Gern geschehen! sowie Hiermit erkläre ich Sie zu Mann und Frau!. Wichtig ist, dass die Verwendung der RF primär durch die Art der Kommunikationssituation bestimmt wird und sie "konventionelle Äußerungsformen für den Vollzug bestimmter Sprechakte, u. U. mit dem Verweis auf ein bestimmtes soziales Ordnungs- und Wertesystem" (Stein 2004: 206) darstellen. ${ }^{1}$

Die beiden ersten einsprachigen deutschen Lernerwörterbücher, das LANGENSCHEIDT GROßWÖRTERBUCH DEUTSCH ALS FREMDSPRACHE (LDaF, 1993) und das WÖRTERBUCH

\footnotetext{
${ }^{1}$ Sie sind abzuheben von den situationsungebundenen oder 'gesprächsspezifischen Formeln', wie z. B. weißt du, offen gesagt, deren Verwendung vorrangig durch eine bestimmte kommunikative Aufgabe motiviert ist (Stein 2004: 266) und die einer getrennten Behandlung bedürfen.
} 
DEUTSCH ALS FREMDSPRACHE von de Gruyter (GDaF, 2000), wurden in den beiden Diskussionsbänden zum LDaF (Barz/Schröder 1996; Wiegand 1998) und einem Diskussionsband zum GDaF (Wiegand 2002) unter anderem auf die Darstellung der RF metalexikografisch besprochen. Die beiden neuesten Erscheinungen im Bereich der einsprachigen Lernerwörterbücher, das PONS GROßWÖRTERBUCH DEUTSCH ALS FREMDSPRACHE (PDaF) (2004) und das WAHRIG GROßWÖRTERBUCH DEUTSCH ALS FREMDSPRACHE (WDaF) (2008), wurden unter Zuhilfenahme des dreißig Fragen umfassenden Kriterienkatalogs (cf. Lipinski 2008) gezielt auf die Darstellung der RF abgetastet (Lipinski im Druck). Leider musste in bislang allen Evaluationen festgestellt werden, dass die Darstellungspraxis zwar etwas variiert, jedoch beständig ein Spalt zwischen phraseologischer Forschung und Wörterbuchpraxis klafft, der auch von den neuesten Erscheinungen bedauernswerterweise nicht verringert wurde.

Vereinzelt wurde in der Literatur zur Lernerlexikografie der RF auch dem Anspruch Rechnung getragen, dass "kein Beitrag [...] bei der kritischen Analyse stehen bleiben" sollte, doch bleiben die Vorschläge häufig im Schatten anderer besprochener Aspekte zurück (Wiegand 1998: IX). Andere Beiträge beschäftigen sich wiederum mit Vorschlägen für die Erstellung eines separaten RF-Wörterbuchs. So wünschenswert ein solches Wörterbuch ist, so werden die meisten Lerner im Alltag dennoch derzeit und weiterhin RF in einem PrintLernerwörterbuch nachschlagen. Aus diesem Grund wird an dieser Stelle der Fokus ganz auf Anregungen und Vorschlägen zur Darstellung der RF im einsprachigen deutschen Lernerwörterbuch in der Printversion liegen. Da grundlegende Anforderungen an eine adäquate Darstellung der RF aus dem Kriterienraster (Lipinski 2008) abgeleitet werden können, werden in diesem Aufsatz nur über das Kriterienraster hinausreichende Überlegungen bezüglich ihrer lexikografischen Darstellung gemacht. Für elektronische Wörterbücher gelten ähnliche Anforderungen. Da sie aber nicht im Druckraum beschränkt sind, ergeben sich für elektronische Wörterbücher unterschiedliche Lösungsmöglichkeiten einiger lexikografischer Herausforderungen. Um dem Gegenstand der RF sowohl in elektronischen Wörterbüchern als auch in Printwörterbüchern gerecht zu werden, sollten diese jeweils individuell besprochen werden. Die hier angestellten Überlegungen zur Darstellung von RF in Printwörterbüchern verstehen sich dabei nicht als endgültige Lösungsangebote, sondern vielmehr als Problemaufriss und Beitrag zur metalexikografischen Diskussion.

\section{2 Überlegungen zu RF in der Rahmenstruktur}

Für die Auswahl der ins Wörterbuch aufgenommenen RF kommt besonders das Kriterium der Auftretenshäufigkeit in Betracht. Korpusbasierte Frequenzanalysen können darüber Aufschluss geben. Wichtig ist hierbei, dass nicht absolute Häufigkeiten sondern relative Häufigkeiten ermittelt werden, denn nur so kann die Relevanz einer RF und die damit verbundene Aufnahme ins Wörterbuch relativ zu anderen RF beurteilt werden. Auch zur Aufnahme der Verwendungsbeispiele zu den RF wird eine am Performanz-Prinzip orientierte Vorgehensweise favorisiert. ${ }^{2}$

Der RF-Suchbereich sollte mit einem einleitenden Symbol versehen werden, z. B. $\boldsymbol{R} \boldsymbol{F}$ für Routineformel, welches darauf hinweist, dass es sich hierbei um eine besondere Gruppe sprachlicher Einheiten handelt, und welches gleichzeitig einen Verweis auf den in den Benutzerhinweisen enthaltenen Erklärungsteil zu den RF darstellt.

Damit der Lerner nicht Gefahr läuft, die RF fehlerhaft zu dekodieren, indem er nicht die ganzheitliche Bedeutung erfasst, sondern aus den einzelnen Konstituenten zu erschließen

\footnotetext{
${ }^{2}$ Da RF reichhaltige Funktionen in der mündlichen Kommunikation haben, sollten Korpora mit großen Anteilen gesprochener Sprache verwendet werden. Den derzeit weit kleineren Korpora gesprochener Sprache im Vergleich zu Korpora geschriebener Sprache ist weiterhin ein Ausbau zu wünschen (cf. Heine 2008).
} 
versucht (Gläser 1990: 130), ist eine einheitliche, die RF als in sich abgeschlossene Aussage kennzeichnende Darstellung notwendig. Bisher ist $\mathrm{m}$. W. noch keine einheitliche Verfahrensweise zur Auswahl des Satzzeichens vorhanden. Eine solche zu finden stellt somit ein Desiderat dar. Eine konsequente Darstellung mit Satzanfangsgroßschreibung und Satzzeichen am Ende der RF wäre hier eine Möglichkeit.

Markierungen im Wörterbuch, die die Verwendungsmöglichkeiten eines Ausdrucks einschränken und näher bestimmen, sind für eine nicht nur grammatisch und semantisch korrekte, sondern auch kommunikativ angemessene Verwendung der jeweils lexikografisch kommentierten Spracheinheiten unerlässlich. Damit die Wörterbuchbenutzer die angewandten Markierungen richtig interpretieren und für sich nutzen können, ist eine Klassifizierung der Markierungen notwendig. Leider ist, wie Bock (2003: 148) feststellt, auch innerhalb der muttersprachlich orientierten Lexikografie des Deutschen für Markierungen kein einheitliches System zu finden, das es $\mathrm{zu}$ übernehmen gäbe. Auch in Beug auf ein einheitliches Markierungssystem sind wir also mit einem Forschungsdesiderat konfrontiert. Eine zu rigorose Orientierung an einmal erstellten theoretischen Kriterien, so Püschel (1988: 491), muss jedoch den sprachlichen Sachverhalten nicht unbedingt gerecht werden. Daher ist es notwendig, bei der Darstellung der im Wörterbuch verwendeten Markierungsstrategien eine einräumende Anmerkung, wie z. B. die von Kempcke im Handwörterbuch der deutschen Gegenwartssprache, zu formulieren:

Die Festlegung schließt jedoch nicht aus, dass ein Sprecher situativ bedingt eine lexikalische Einheit auf einer anderen als der im Wörterbuch markierten Ebene adäquat verwenden kann.

(Kempcke 1994: XXII)

Generell ist zudem in den Benutzerhinweisen ausdrücklich darauf hinzuweisen, dass es sich bei den RF um eine Gruppe stark situationsabhängiger sprachlicher Einheiten handelt. Durch die Mehrfachkodierung vieler RF werden diese häufig erst durch die Intonation oder Betonung im Situationskontext eindeutig, so z. B. bei Macht nichts! und Sag das noch einmal!. Für den Lerner bedeutet die Mehrfachkodierung insbesondere dann beträchtliche Schwierigkeiten, wenn er mit den für die RF semantisch relevanten Betonungs- und Intonationsunterschieden nicht vertraut ist (cf. Gläser 1990: 130). Sprechaktsequenzen und die damit z.T. korrelierenden intonatorischen Mittel sollten daher in den Benutzungshinweisen erläutert werden. Für den Wörterbuchbenutzer ist es insgesamt von großer Wichtigkeit, dass alle bisherigen wie auch die im Folgenden beschriebenen Vorgehensweisen, vor allem der Gebrauch verschiedener Zeichen und Symbole, in den Außentexten des Wörterbuchs mit Hilfe von Beispielen erklärt werden.

\section{3 Überlegungen zu RF in der Mikro- und Mediostruktur}

\section{1 Überlegungen zur Auffindbarkeit und Nennformschreibung}

Die schnellstmögliche Auffindbarkeit der gesuchten Information ist im Interesse aller Wörterbuchbenutzer. Hierzu ist eine in den Rahmentexten erläuterte und konsequent umgesetzte Auflistung der RF-Einträge unter den Lemmata des Wörterbuchs Voraussetzung. $\mathrm{Zu}$ den Wesensmerkmalen der RF gehört, dass sie syntaktisch höchst unterschiedlich sind. Das erschwert es, sie immer unter einer bestimmten Komponente aufzulisten. Bei RF mit expliziter Satzstruktur lassen sich möglicherweise Substantiv oder Verb als Zuordnungspunkte finden, bei RF mit impliziter Satzstruktur, z. B. Bis dann! oder Hand aufs Herz!, die bar einer substantivischen und/oder verbalen Komponente sind, wird die Schwierigkeit einer Auflistung nach syntaktischen Kriterien deutlich. Eine Möglichkeit, dem zu begegnen besteht darin, das Alphabetisierungsschema von Schemann (1993: XVIf.) wie folgt an die RF anzupassen: 


\begin{tabular}{|l|c|c|c|c|}
\hline $\begin{array}{l}\text { Reihenfolge der Beachtung } \\
\text { der Komponenten }\end{array}$ & \multicolumn{4}{|c|}{$\begin{array}{l}\text { Komponente vorhanden } \\
(+)=\text { ja; (-)= nein }\end{array}$} \\
\hline 1. Substantiv & + & - & - & - \\
\hline 2. Verb & & + & - & - \\
\hline 3. Adjektiv & & & + & - \\
\hline 4. Adverb & & & & + \\
\hline
\end{tabular}

Tabelle 1: Für RF angepasstes Alphabetisierungsschema

Enthält die RF ein Substantiv, so bestimmt dieses die Einordnung. Enthält die RF kein Substantiv, sondern ein Verb, so bestimmt dieses die Einordnung etc., sodass sich die Abfolge Substantiv-Verb-Adjektiv-Adverb ergibt. Herbst/Klotz beschreiben eine Einordnung nach dem Alphabetisierungsprinzip als "unmittelbar nachvollziehbar und insofern äußerst benutzerfreundlich" (Herbst/Klotz 2003: 189). Um jedoch die bestmögliche Auffindbarkeit zu gewährleisten, müssten die RF unter allen enthaltenen verbalen und substantivischen oder gegebenenfalls anderen Komponenten verzeichnet sein. Diese Vorgehensweise würde den Wörterbuchumfang enorm anschwellen lassen. Um diesem Problem Rechnung zu tragen, kann statt einer Auflistung unter allen Komponenten ein Register und eine angemessene Verweisstruktur eingerichtet werden. Für Kollokationen hängt eine Platzierung unter Substantiv oder Verb, so Bahns (1993: 144), von der Benutzungssituation, Textproduktion oder -rezeption, ab. Diese Feststellung kann m. E. für die RF übernommen werden. Lediglich für rezeptive Zwecke erscheint eine Einordnung unter dem Lemma einer oder mehrerer enthaltener Komponenten sinnvoll. Für produktive Zwecke ist diese Art der Einordnung "für den lexikographischen Zopf, den es abzuschneiden gilt" (Kühn 1994: 422) zu halten. Eine lemmatypische und onomasiologisch-distinktive Beschreibung von RF im Rahmen sowohl lexikalischer als auch phraseologischer Verwandtschaft sollte für produktive Zwecke favorisiert werden.

Es stellt sich weiterhin die Frage, an welcher Stelle innerhalb des jeweiligen Artikels der Eintrag der RF erfolgen sollte. In Anbetracht der Tatsache, dass RF auf Grund der Eigenschaften Festigkeit und Polylexikalität den Phraseologismen zuzuordnen sind, sind sie m. E. innerhalb eines phraseologischen Suchbereichs anzusiedeln. Da sie sich jedoch hinsichtlich ihrer Zeichenfunktion stark von den restlichen Phraseologismen unterscheiden, sollte ihnen ein gesonderter Abschnitt innerhalb des phraseologischen Suchbereichs zugewiesen werden. ${ }^{3}$ Da auch bestimmte Einworteinheiten zur Gruppe der RF gehören, z. B. Hallo!, Tschüss!, kommt für die RF einerseits eine Auflistung am Anfang des phraseologischen Suchbereichs in Frage. Andererseits kommt aufgrund der meist geringen referentiellen Bedeutung der RF ebenfalls ein Eintrag am Ende des phraseologischen Suchbereichs in Betracht. Auf diese Weise könnte darauf aufmerksam gemacht werden, dass RF überwiegend kommunikative Funktionen innehaben. Die Auflistung mehrerer RF innerhalb des RF-Suchbereichs sollte einer zuvor festgelegten Ordnung folgen. Zwei oder mehr RF, die dasselbe alphabethische Leitwort haben, können über die übrigen Konstituenten erneut nach dem Alphabetisierungsschema in eine Reihenfolge gebracht werden. Eine andere Möglichkeit ist, sie nach den unterschiedlichen Funktionen, die sie bei der Herstellung, Definition, dem Vollzug und der Beendigung kommunikativer Handlungen übernehmen, zu ordnen. Eine interne Untergliederung nach den fünf Sprechakttypen, die durch die Äußerung einer RF vollzogenen werden können, ist ebenfalls denkbar. Für die RF käme zur Bestimmung der Reihenfolge innerhalb des RF-Suchbereichs zudem das Kriterium der

\footnotetext{
${ }^{3}$ Dieser könnte, wie bereits im Abschnitt zur Rahmenstruktur erläutert, mit einer einleitenden Markierung, z. B. $\boldsymbol{R} \boldsymbol{F}$, versehen werden.
} 
Auftretenshäufigkeit in Betracht. Wörterbuchbenutzer erhielten durch diese Art der Aufreihung Informationen zur Häufigkeit des Vorkommens einer RF, relativ zu den anderen, die im gleichen Eintrag aufgelistet werden, z. B. Grüß Gott! vor Gott zum Gruß!. ${ }^{4}$ Wie sich diese Reihenfolge auf die Benutzerfreundlichkeit auswirkt, ist zu überprüfen.

\section{2 Überlegungen zu semantischen und pragmatischen Informationen}

Eine wünschenswerte Bedeutungsbeschreibung erfolgt mittels einer Kombination aus freien Wortverbindungen satzsynonymischer Art, durch pragmatische Kommentare, die Sprechaktpräferenzen und -restriktionen erhellen, und unter Verwendung von authentischen Beispielen, auf mehreren Ebenen geben werden. Pragmatische Markierungen, die den präferierten oder begrenzten Gebrauch lexikalischer Einheiten, bezogen auf verschiedene Gruppen von Sprechern, Stilebenen, Situationen, Zeiten u. a. verdeutlichen sollen, werden in Wörterbüchern häufig nur unzureichend strukturiert und intuitiv gesetzt. Sie sind demgemäß vage und nicht widerspruchsfrei (cf. Lerchner 1996: 133). Ein einheitliches System zur Markierung lexikalischer Einheiten, in diesem Fall der RF, ist zu erstellen. Ein solches System sollte sich jedoch durch flexible Grenzen auszeichnen, um eine genügende und zugleich anschauliche Präsentation im Wörterbuch zu ermöglichen. Fach- und Gruppensprachzugehörigkeiten, stark überwiegende Zugehörigkeit zu gesprochener vs. geschriebener Sprache und diatopische Markierungen sind durch geeignete Korpusanalysen zu ermitteln. Diatopische Markierungen sollten dabei möglichst differenziert, z. B. mit nordost-, süddeutsch, österreichisch, schweizerdeutsch etc., und nicht undifferenziert wie durch regional gemacht werden. Für zeitliche Markierungen wären die Markierungen veraltet oder veraltend denkbar, insofern derartig $\mathrm{zu}$ markierender Wortschatz überhaupt in ein Lernerwörterbuch der Gegenwartssprache aufgenommen wird.

Zur Markierung des durch die Äußerung einer RF vollzogenen Sprechakts wäre eine Markierung direkt an der aufgeführten RF, in Form eines hochgestellten Buchstabens, der die Abkürzung des jeweiligen Sprechakttyps darstellt, vorstellbar. Mehrfachmarkierungen jener RF, die verschiedene Sprechakte darstellen können, wären dabei ausdrücklich erwünscht. Die Reihenfolge der aufzulistenden verschiedenen Verwendungsweisen einer RF kann $\mathrm{m}$. E. durch die Verwendungshäufigkeit als jeweiliger Sprechakttyp bestimmt werden. Als Erstes würde der jeweils durch Einsatz der RF meistvollzogene Sprechakttyp genannt werden. Zur Ermittlung der Häufigkeiten bieten sich pragmatische Frequenzanalysen mittels elektronischer Korpora an.

Ein Vorschlag von Cheon (1998: 61-83) bezüglich der Darstellung von Phraseologismen im zweisprachigen Lernerwörterbuch geht dahin, nach Nennform sowie Bedeutungs- und Beispielangaben eine "Mikropragmatik" (ebd.: 93) aufzuführen. Cheon schlägt dazu die pragmatischen Parameter Intention, Emotionaler Gehalt, Vertrautheit der Gesprächspartner, Transaktionsebene und Anwendungsbereich, vor.5 Für Wotjak (2005) "besteht kein Zweifel daran, dass die vorgeschlagenen fremdsprachlerfreundlichen Verfeinerungen der Mikrostrukturbeschreibung die Gebrauchssicherheit erhöhen" (Wotjak 2005: 381). M. E. ist der Vorschlag Cheons besonders für die Gruppe der RF interessant, sollte jedoch noch ergänzt werden durch die oben beschriebenen Markierungen $\mathrm{zu}$ Fach- und Gruppensprachzugehörigkeit, stark überwiegende Zugehörigkeit zu gesprochener vs. geschriebener Sprache, diatopische Markierungen sowie Informationen zur Intonation und

\footnotetext{
4 Wahrig (1983: 19) schlägt dieses Vorgehen generell für die Darstellung der Bedeutungen unter einem Lemma vor.

5 Angaben zu grammatischen Besonderheiten werden von Cheon (1998: 78) ebenfalls der Pragmatik zugeordnet. Für die Lexikografie macht es jedoch Sinn, sie der Nennformschreibung, d. h. vielmehr der grammatischen Ebene, zuzuordnen. Aus diesem Grund werden diese Parameter hier nicht mit aufgelistet.
} 
eine Kennzeichnung der Möglichkeit humoristischen Sprachgebrauchs. Auch ist dem System Cheons noch eine Lösung für die Kennzeichnung von unterschiedlichen Verwendungsmöglichkeiten hinzuzufügen.

Phonetisch-phonologische Angaben, die Information über die Akzentuierung der RF und zur Vokalquantität innerhalb der RF geben können, können im Printwörterbuch durch Punkte unter den kurzen Vokalen bzw. Striche für lange Vokale im akzentuierten Wort gemacht werden. Für den Fall, dass die RF eine Interjektion beinhaltet, werden zwei Akzentuierungen angegeben, da Interjektionen immer betont sind (cf. Stock 1996: 57). Hirschfeld (1996) macht darauf aufmerksam, dass phonetische Fehler oftmals durch "Unsicherheiten in der Anwendbarkeit" (Hirschfeld 1996: 32) entstehen. Daher sollten Informationen zur Intonation innerhalb der Makro- und Mikrostruktur nie getrennt von den pragmatischen Angaben gemacht werden.

\section{3 Überlegungen zu Beispielen, zum Netz von Verweisangaben und zur graphischen Beschaffenheit der Artikelstruktur}

So häufig bisher die Rede von Verdeutlichung durch Beispiele war, so dringend gilt es, in Bezug auf RF zu klären, was unter einem guten Beispiel zu verstehen ist. Denn wie angemessene Beispiele zu RF konkret auszusehen haben, steht $\mathrm{m}$. W. noch zur Untersuchung aus. Anforderungen an Beispiele sind in jedem Falle die Ergänzung der in der Bedeutungsangabe gemachten Aussage durch die Vorführung eines in eine Situation eingebetteten, kontextangemessenen und textsortenspezifischen Verwendungsbeispiels. Mehrere Beispiele sollten gemacht werden, wenn verschiedene Verwendungsweisen einer RF möglich sind. Die Angabe mehrerer unterschiedlicher, kontextuell eingebundener Beispiele vermag dazu beizusteuern, den Benutzer für kontextsensitive Bedeutungen zu sensibilisieren.

Durch die Verweisstruktur sollte zum einen intensiv auf andere Wörterbucheinträge verwiesen werden, vor allem dann, wenn die RF nicht unter allen enthaltenen Komponenten gelistet werden. Zum anderen sollten Verweise auf die onomasiologisch gegliederten Übersichtsbereiche oder auch auf eventuell enthaltene Bereiche mit Angaben für produktive Zwecke gemacht werden. Verweise und Artikelstruktur sollten im Lernerwörterbuch so beschaffen sein, dass sie übersichtlich, mit klarem Layout und durchgängiger Systematik gestaltet sind. Dazu sollte auf den Einsatz von Abkürzungen weitgehend verzichtet werden. Deutliche Signalsymbole und/oder grafische Mittel zur Abhebung sowohl des phraseologischen Suchbereichs, als auch der darin eingegliederten RF sollten angestrebt werden. Die Einheitlichkeit der Darstellungen, Bedeutungskommentierungen, der Lemmatisierung einer RF unter unterschiedlichen Komponenten etc. sowie eine Einheitlichkeit in allen Strukturteilen des Wörterbuchs sollten wichtige Prinzipien darstellen.

\section{$4 \quad$ Zusammenfassung}

An dieser Stelle werden die Überlegungen zur Darstellung der RF im einsprachigen Lernerwörterbuch noch einmal zusammengefasst.

- Die Prägung der RF durch ihre kommunikative Funktion ist für den Wörterbuchbenutzer zu erläutern. Dabei ist auch explizit auf mögliche pragmatische Mehrfachkodierungen der RF hinzuweisen. Es sollte für den Stellenwert einer korrekten Verwendung sowie möglicherweise den Vollzug von Wertungen durch die Verwendung dieser sprachlichen Einheiten sensibilisiert werden.

- Die Relevanz von Gebrauchsangaben zu den RF, um eine korrekte Verwendung dieser zu gewährleisten, wurde auch in dieser Arbeit als Notwendigkeit herausgearbeitet. Strukturierende und zugleich flexible pragmatische Markierungssysteme, die 
zusätzlich zu den bisher beachteten Parametern auch Intention, Status, Vertrautheitsebene und emotionalen Gehalt der Gesprächsteilnehmer berücksichtigen, sind zu erstellen.

- Betonungs- und Intonationsmuster sind in die Gebrauchsangaben und Bedeutungserklärungen stärker einzubeziehen.

- RF sollten als abgeschlossene Aussagen markiert werden.

- Kommunikative Funktion und Stellenwert der RF sind u. a. auch Gründe dafür, einen eigenen Suchbereich für RF innerhalb des phraseologischen Suchbereichs als einrichtenswert zu erachten. Ein ausgeprägtes Netz von Verweisangaben zu den RF ist, nicht allein um Druckraumbeschränkungen ernst zu nehmen, im Wörterbuch einzurichten.

- Eine Einbeziehung korpuslinguistischer Möglichkeiten ist wünschenswert in Bezug auf Bedeutung und Bedeutungsvarianten, Beispielgewinnung, pragmatische Besonderheiten wie Sprechaktbestimmungen, Gebrauchsüblichkeiten und Restriktionen sowie Häufigkeitsrelationen zur Auswahl der RF für das Wörterbuch, Reihenfolgeerstellung, Verwendungskontexte u. a.

- Initialalphabetische Einordnungen der RF in die Makrostruktur, nach Komponenten der RF, sind durch lemmatypische Einordnungen und onomasiologische Suchbereiche zu ergänzen.

- Komplementär semasiologisch-onomasiologische Wörterbücher kämen einer funktional orientierten Darstellung der RF entgegen.

Generell ist eine Intensivierung der Beachtung von RF im allgemeinen Lernerwörterbuch wünschenswert. Zusätzlich ist selbstverständlich die Erstellung von Spezialwörterbüchern für RF für die einsprachige und zweisprachige Wörterbuchlandschaft mit Deutsch anzustreben. Die Erkenntnisse, die durch die Forschungsbemühungen in Bezug auf die Erstellung eines RF-Spezialwörterbuchs gewonnen werden, können und sollen dann wiederum unbedingt in die Verbesserung der allgemeinen einsprachigen Lernerwörterbücher einfließen.

\section{Literatur}

Bahns, Jens (1993): "Wer eine günstige Gelegenheit verpasst, kann beträchtlichen Schaden davontragen. Kollokationen in Langenscheidts Großwörterbuch Deutsch als Fremdsprache". Lernen in Deutschland 21: 137-155.

Barz, Irmhild/Schröder, Marianne (eds.) (1996): Das Lernerwörterbuch Deutsch als Fremdsprache in der Diskussion. Heidelberg: Winter.

Bock, Ulrich (2003): Die "verhüllende" Bedeutungserklärung. Versuch einer Neuordnung der stilistischen Markierungen im einsprachigen Wörterbuch der deutschen Gegenwartssprache. Frankfurt a. M.: Lang.

Burger, Harald (2007): Phraseologie. Eine Einführung am Beispiel des Deutschen. 3., neu bearb. Aufl. Berlin: Schmidt. (= Grundlagen der Germanistik 36).

Cheon, Mi-Ae (1998): Zur Konzeption eines phraseologischen Wörterbuchs für den Fremdsprachler. Tübingen: Niemeyer.

GDaF = Kempcke, Günter (2000): Wörterbuch Deutsch als Fremdsprache. Berlin: de Gruyter.

Gläser, Rosemarie (1990): Phraseologie der englischen Sprache. 2. unveränd. Aufl. Leipzig: Enzyklopädie.

Heine, Antje. (2008): "Zur Nutzbarkeit der gegenwärtig verfügbaren deutschen Korpora für die Lernerlexikografie Deutsch als Fremdsprache. Anspruch und Wirklichkeit". Deutsch als Fremdsprache 44: 3-8. 
Herbst, Thomas/Klotz, Michael (2003): Lexikografie. Paderborn: Schöning. (= UTB 8263). Hirschfeld, Ursula (1996): "Der Ton spielt die Musik". Fremdsprache Deutsch 15: 31-35.

Kempcke, Günter (1994): "Zur Darstellung der kommunikativen Wendungen in den gegenwartssprachlichen Wörterbüchern des Deutschen". In: Sandig, Barbara (ed.): EUROPHRAS 92. Tendenzen der Phraseologieforschung. Bochum, Brockmeyer: 303-314.

Kühn, Peter (1994): "Pragmatische Phraseologie: Konsequenzen für die Phraseographie und Phraseodidaktik". In: Sandig, Barbara (ed.): EUROPHRAS 92. Tendenzen der Phraseologieforschung. Bochum, Brockmeyer: 411-428.

LDaF = Götz, Dieter/Haensch, Günther/Wellmann, Hans (eds.) (2003): Langenscheidt Großwörterbuch Deutsch als Fremdsprache. Das einsprachige Wörterbuch für alle, die Deutsch lernen. Neubearbeitung. Berlin: Langenscheidt.

Lerchner, Gotthard (1996): "Informationen über die kulturspezifisch-pragmatische Markiertheit von lexikalischen Ausdrücken im Lernerwörterbuch". In: Barz, Irmhild/Schröder, Marianne (eds.): Das Lernerwörterbuch Deutsch als Fremdsprache in der Diskussion. Heidelberg, Winter: 129-146.

Lipinski, Silke (2008): "Routineformeln im Lernerwörterbuch DaF. Ein Kriterienkatalog". Deutsch als Fremdsprache 45: 91-98.

Lipinski, Silke (im Druck): "Zu Routineformeln im einsprachigen deutschen Lernerwörterbuch: eine Untersuchung anhand von zwei Lernerwörterbüchern". In: Finnische Beiträge zur Germanistik.

PDaF = Cyffka, Andreas (ed.) (2006/2008): PONS Großwörterbuch Deutsch als Fremdsprache. Stuttgart: Klett. [Erscheinungsjahr der Publikation ist das Jahr 2008, es wurde in der Publikation aber fälschlicherweise das Erscheinungsjahr 2006 angegeben; Quelle: Onlinekatalog der Deutschen Nationalbibliothek].

Püschel, Ulrich (1988): "Zu Status und Funktion arealer Kennzeichnungen in allgemeinen einsprachigen Wörterbüchern". In: Munske, Horst H. et al. (eds.): Deutscher WortschatzLexikologische Studien. Berlin: de Gruyter.

Schemann, Hans (1993): Deutsche Idiomatik. Die deutschen Redewendungen im Kontext. Stuttgart: Klett.

Stein, Stephan (2004): "Formelhaftigkeit und Routinen in mündlicher Kommunikation". In: Kathrin Steyer (ed.): Wortverbindungen - mehr oder weniger fest. Berlin, de Gruyter: 262288 (= IDS Jahrbuch 2003).

Stock, Eberhard (1996): Deutsche Intonation. Leipzig: Langenscheidt.

Wahrig, Gerhard (1983): "Neue Wege in der Wörterbucharbeit". In: Wahrig, Eva (ed.): Gesammelte Schriften. Gerhard Wahrig. Tübingen: Narr.

WDaF = Wahrig-Burfeind, Renate (2008): WAHRIG Großwörterbuch Deutsch als Fremdsprache. Gütersloh/München: Wissen Media.

Wiegand, Herbert Ernst (ed.) (1998): Perspektiven der pädagogischen Lexikographie des Deutschen: Untersuchungen anhand von "Langenscheidts Großwörterbuch Deutsch als Fremdsprache". Tübingen: Niemeyer. (= Lexicographica. Series Maior 86).

Wiegand, Herbert Ernst (ed.) (2002): Perspektiven der pädagogischen Lexikographie des Deutschen: Untersuchungen anhand des "de Gruyter Wörterbuchs Deutsch als Fremdsprache". Tübingen: Niemeyer. (= Lexicographica. Series Maior 110).

Wotjak, Barbara (2005): "Routineformeln im Lernerwörterbuch". In: Irmhild Barz/Henning Bergenholtz/Jarmo Korhonen (eds.): Schreiben, Verstehen, Übersetzen, Lernen. Zu einund zweisprachigen Wörterbüchern mit Deutsch. Frankfurt a. M., Lang: 371-388. (= Finnische Beiträge zur Germanistik 14). 